\title{
Evidence for a new paradigm of gonorrhoea transmission: cross-sectional analysis of Neisseria gonorrhoeae infections by anatomical site in both partners in 60 male couples
}

\author{
Vincent Jasper Cornelisse, ${ }^{\oplus 1,2,3,4}$ Deborah Williamson, ${ }^{5}$ Lei Zhang, ${ }^{\bullet}$ 1,3,6 \\ Marcus Y Chen, ${ }^{1,3}$ Catriona Bradshaw, ${ }^{1,3}$ Jane S Hocking, ${ }^{7}$ Jennifer Hoy, ${ }^{3,4}$ \\ Benjamin P Howden, ${ }^{5,8}$ Eric P F Chow, ${ }^{1,3}$ Christopher K Fairley ${ }^{1,3}$
}

\begin{abstract}
- Additional material is published online only. To view please visit the journal online (http://dx.doi.org/10.1136/ sextrans-2018-053803).

For numbered affiliations see end of article.
\end{abstract}

\section{Correspondence to} Dr Vincent Jasper Cornelisse, Melbourne Sexual Health Centre, Carlton, VIC 3053, Australia; vcornelisse@mshc. org.au

EPFC and CKF are joint senior authors.

These data were presented as oral abstracts at the IUSTI World Congress in Dublin in June 2018 and the IUSTI AsiaPacific Congress in Auckland in November 2018.

Received 12 August 2018 Revised 12 February 2019 Accepted 3 March 2019 Published Online First 17 April 2019

\section{Linked}

- http://dx.doi.org/10.1136/ sextrans-2018-053911

- http://dx.doi.org/10.1136/ sextrans-2018-053957

- http://dx.doi.org/10.1136/ sextrans-2019-054030

\section{Check for updates}

(c) Author(s) (or their employer(s)) 2019. No commercial re-use. See rights and permissions. Published by BMJ.

To cite: Cornelisse VJ, Williamson $D$, Zhang $\mathrm{L}$, et al. Sex Transm Infect 2019:95:437-442.

\section{ABSTRACT}

Objective Gonorrhoea transmission between men is currently thought to occur primarily to and from the urethra. Transmission without urethral involvement, from throat-to-throat and throat-to-anus, is considered to be uncommon. Using gonorrhoea results from male couples, we aimed to investigate the transmission dynamics of gonorrhoea. If current medical consensus is correct, then most throat and anal infections should be explained by the partner's urethral infection.

Methods This is a cross-sectional analysis of gonorrhoea diagnosed by nucleic acid amplification tests in both partners in male couples who attended Melbourne Sexual Health Centre together between March 2015 and June 2017. Isolates obtained from culture-positive infections underwent whole genome sequencing to assess phylogenetic relatedness between partners.

Results In all 60 couples (120 men) at least one partner had gonorrhoea, and isolates had very high phylogenetic relatedness between partners. After excluding men with urethral gonorrhoea, among 32 men with anal gonorrhoea, $34 \%(95 \% \mathrm{Cl} 19 \%$ to $53 \%)$ had a partner with throat gonorrhoea. After excluding couples where either man had urethral gonorrhoea, among 48 couples in which at least one man had throat gonorrhoea, in $23 \%(95 \% \mathrm{Cl} 12 \%$ to $37 \%)$ of couples both men had throat gonorrhoea.

Conclusions The observed gonorrhoea positivity when urethral infection is absent supports a new paradigm of gonorrhoea transmission, where the throat is a major source of gonorrhoea transmission between men, through tongue kissing, oroanal sex and saliva use as anal lubricant. Public health messages may need to address the risk of saliva exposure during sex.

\section{INTRODUCTION}

In recent years, Neisseria gonorrhoeae (gonorrhoea) diagnoses have risen sharply among gay, bisexual and other men who have sex with men (MSM) in many countries including Australia and the USA. ${ }^{12}$ Increasing prevalence is a potential driver of antimicrobial resistance in N. gonorrhoeae, ${ }^{3}$ and in response the WHO and the US Centers for Disease
Control and Prevention have called for enhanced efforts to control gonorrhoea. ${ }^{4}$

Current public health strategies are based on the premise that gonorrhoea transmission between men occurs primarily during condomless penoanal sex, with a lesser role for oropenile sex ('fellatio'). In other words, the urethra plays a central role in the conventional paradigm of gonorrhoea transmission among MSM (online supplementary figure 1A). However, urethral gonorrhoea is usually symptomatic, ${ }^{67}$ symptoms usually appear within days, ${ }^{7}$ and in countries with accessible healthcare most cases are treated expeditiously and hence have limited opportunity to contribute to onward transmission. ${ }^{78}$ To explain the high prevalence of gonorrhoea, Fairley et $a l^{9}$ proposed a paradigm where incident gonorrhoea among MSM is driven mainly by transmission between throats (ie, during tongue kissing) with onward transmission by saliva to partners' anus and/or urethra during oroanal and oropenile sex, respectively (online supplementary figure $1 \mathrm{~B}) .{ }^{9}$ Thus, the urethra is primarily a recipient of infection, and not a major source of onward transmission.

More than 40 years ago, case reports described gonorrhoea transmission by kissing. ${ }^{10} 11$ However, observational studies have not identified kissing as an independent risk factor for throat gonorrhoea. ${ }^{12} 13$ One significant problem is the strong collinearity between different sexual practices. In other words, when men have sex with men, they often have oropenile, oroanal and penoanal sex, and they kiss. ${ }^{13}$ This makes it difficult to attribute the risk of gonorrhoea to an individual sexual activity. Another significant problem in these observational studies is the lack of data on tongue kissing with partners with whom men do not have sex ('kissing-without-sex' partners), particularly when one considers that MSM tend to have similar numbers of 'kissing-without-sex' partners as they do 'kissing-with-sex' partners. ${ }^{9} 14$

As an alternative methodology, rather than assess associations with different sexual practices, we assessed anatomical site-specific gonorrhoea infections in both partners in male couples, from which we aimed to infer the likelihood of gonorrhoea 
transmission between partners' throat and anus, and between throats. We then assessed whether these data support the conventional paradigm of gonorrhoea transmission, in which urethral gonorrhoea plays a central role, or whether they instead support the new paradigm proposed by Fairley et $a l^{9}{ }^{9}$ in which throat gonorrhoea is the central driver of transmission. If the conventional paradigm is correct, then one would expect most throat and anal infections to be explained by urethral infection in a man's partner. If the new paradigm is correct, then one would expect concurrent throat-and-throat gonorrhoea and throatand-anus gonorrhoea between partners to persist after excluding couples with urethral gonorrhoea.

\section{METHODS}

This was a cross-sectional retrospective analysis of routinely collected clinic data, in which we analysed positivity of gonorrhoea diagnosed by nucleic acid amplification testing (NAAT) in both partners in male couples who together attended Melbourne Sexual Health Centre (MSHC) between 25 March 2015 and 29 June 2017. MSHC is the only public sexual health clinic in Melbourne, Australia, and provides free walk-in service. During the study period, MSHC's general clinic provided 44000 consultations annually (21 000 MSM).

\section{Participant eligibility and data collection}

All patients were routinely asked to complete a computer-assisted self-interview (CASI) covering demographics and sexual practices, including their number of partners and frequency of condom use for penoanal sex in the preceding 3 months. ${ }^{15}$ The CASI also asked 'Is your partner being seen today at this clinic?' and asked for their partner's name. We included all men whose male partner we were able to identify as a patient attending on the same day.

All MSM patients were routinely offered screening for throat, anal and urethral gonorrhoea by NAAT, regardless of symptoms or a history of exposure, in accordance with Australian guidelines. ${ }^{16}$ In addition, symptomatic patients and patients who returned for treatment after positive NAAT were tested by culture on anal, throat and/or urethral swabs.

We included only couples where both men had gonorrhoea NAAT results available for all three anatomical sites (throat, anus and urethra) and where at least one partner tested positive for gonorrhoea.

\section{Laboratory testing}

NAAT testing for N. gonorrhoeae was conducted using the Aptima Combo 2 assay with diagnostic confirmation by the Aptima GC assay (Hologic Panther platform, USA). Culture isolates were confirmed as N. gonorrhoeae using a MALDI Biotyper (Bruker Daltonik, Bremen, Germany). For couples where both men had a culture isolate available, whole genome sequencing (WGS) and bioinformatic analysis were performed. In brief, WGS was performed using Illumina libraries and protocols (Illumina, San Diego, California, USA). Read mapping and alignment was performed to identify single nucleotide polymorphisms (SNPs), and a maximum likelihood phylogeny was inferred from core genome SNPs after filtering for recombination. In silico multilocus sequence typing (MLST) and in silico N. gonorrhoeae multiantigen sequence (NG-MAST) were performed on de novo assemblies. Further details of the bioinformatic analysis are provided in the online supplementary methods.

\section{Data analysis}

There are nine possible combinations or 'pairs' of anatomical sites within couples (online supplementary figure 2). For pairs of different anatomical sites, such as between the throat of one partner and the anus of the other partner, there are twice as many possible pairs (ie, throat-and-anus and anus-and-throat) as there are pairs of the same anatomical site (eg, throat-andthroat). For this reason, for pairs of different anatomical sites (eg, throat gonorrhoea in the partners of men with anal gonorrhoea), we defined the denominator as the number of men who had gonorrhoea at the site of interest (eg, anus), and the numerator as the number of these men whose partner had gonorrhoea at the site of interest (eg, throat). Whereas for pairs of the same anatomical site (eg, throat gonorrhoea in the partners of men with throat gonorrhoea), we defined the denominator as the number of couples in which either man had gonorrhoea at the site of interest (eg, throat), and the numerator as the number of these couples in which both men had gonorrhoea at the site of interest (eg, throat).

We then assessed gonorrhoea positivity in these couples that could not be the result of transmission from urethral gonorrhoea, by removing couples containing men with urethral gonorrhoea from the analyses. For example, we analysed throat gonorrhoea positivity in the partners of men with anal gonorrhoea, after removing couples in which men with anal gonorrhoea also had urethral gonorrhoea. We then again analysed throat gonorrhoea positivity in the partners of men with anal gonorrhoea, but this time after removing couples in which men with throat gonorrhoea also had urethral gonorrhoea.

For pairs of the same anatomical site, we then excluded couples if both men in the couple had urethral gonorrhoea. It is important to note that for pairs of the same anatomical site, we did not exclude a couple if only one man in the couple had urethral gonorrhoea, because, in order for urethral gonorrhoea to be the source of throat or anal gonorrhoea in both men in a couple, both men would need to have urethral gonorrhoea.

We also reviewed results from urethral gonorrhoea tests performed in the 3 months preceding the couples' attendance to see if any of the participants had been diagnosed with urethral gonorrhoea prior to their attendance as a couple, and hence to check whether current throat or anal gonorrhoea could have resulted from a previous episode of urethral gonorrhoea in the partner. We found that none of the participants had been diagnosed with urethral gonorrhoea in the preceding 3 months, and hence no further analyses of these data were required.

We calculated 95\% CI around gonorrhoea positivity, using 'exact' binomial distribution, ${ }^{17}$ to enable comparison of gonorrhoea positivity between the analyses in which couples containing men with urethral gonorrhoea were included and the analyses in which couples containing men with urethral gonorrhoea were excluded. We also compared these data with the background prevalence of gonorrhoea in our broader sample, which we defined as gonorrhoea positivity in men who were in couples where both men completed full three-site testing for gonorrhoea and where neither man had urethral gonorrhoea. The reason for excluding couples with urethral gonorrhoea from this prevalence calculation was because most men with urethral gonorrhoea presented to the clinic with symptoms, and hence these infections represented incident rather than prevalent infections.

We conducted restricted analyses of men who reported no casual sexual partners (CSPs) to explore whether couples where both men had gonorrhoea could have been a result of both partners having contracted gonorrhoea from external partners rather than as a result of within-couple transmission. In these analyses, the denominator was defined as the number of men with gonorrhoea at the site of interest (eg, throat) and who reported having had no CSPs in the preceding 3 months, and the numerator as the number of these 


\begin{tabular}{lll}
\hline Table 1 & Participant characteristics & \\
\hline & 630 men in broader sample & $\begin{array}{l}120 \text { men in couples with } \\
\text { gonorrhoea }\end{array}$ \\
\hline Age & Median 30 years (IQR 25-36) & Median 30 years (IQR 25-36) \\
HIV-positive & $25(4 \%)$ & $10(8 \%)$ \\
Condom use & & \\
\multicolumn{1}{|l}{ IAl } & $93(15 \%)$ used consistently & $10(8 \%)$ used consistently \\
RAI & $92(15 \%)$ used consistently & $10(8 \%)$ used consistently \\
Casual sexual partners & Median 1 (IQR 0-3) & Median 3 (IQR 0-5) \\
\hline
\end{tabular}

Condom use is defined as consistent use of condoms for penoanal sexual intercourse with their regular partner, stratified by insertive and receptive anal intercourse.

Casual sexual partners are defined as the number of sexual partners in the preceding 3

months with whom men had sexual contact in addition to their regular partner.

IAl, insertive anal intercourse; RAl, receptive anal intercourse.

men who had a partner with gonorrhoea at their site of interest (eg, anus).

Within-couple condom use can potentially affect within-couple gonorrhoea concurrency. However, we found that the use of condoms for sexual contact within couples was very low, and hence we did not adjust for condom use in our analysis.

Statistical analyses were performed using STATA V.13.1.

\section{RESULTS \\ Demographics, condom use and gonorrhoea positivity of individual men}

During the study period, 417 couples (834 men) presented together. In 315 couples ( 630 men) both men had tests for gonorrhoea at all three sites (urethra, anus and throat), and in 60 couples (120 men) at least one man in each couple tested positive for gonorrhoea (online supplementary figure 3). These 60 couples form the basis for this analysis.

These 120 men had a median age of 30 years (IQR 25-36), and 10 men had previously been diagnosed with HIV (table 1 ). Eightyfive men had gonorrhoea, of whom 63 had throat gonorrhoea, 48 had anal gonorrhoea and 25 had urethral gonorrhoea; many had multisite gonorrhoea (figure 1). Of the 25 men with urethral gonorrhoea, $22(88 \%)$ were symptomatic, with a median duration of symptoms of 3 days (IQR $1-4$ days).

Among the 120 men, condoms were used consistently by 10 men (8\%) for insertive penoanal sex and by 10 men (8\%) for receptive penoanal sex with their regular partner in the preceding 3 months. A further 12 men (10\%) reported not practising insertive penoanal sex and 13 men (11\%) reported not practising receptive penoanal sex with their partner. The others used condoms inconsistently or never in the preceding 3 months.

To illustrate background gonorrhoea prevalence at our clinic, we present gonorrhoea diagnoses in our broader data set that included 315 couples (630 men) with complete testing for gonorrhoea. We excluded 23 couples where one or both partners had urethral gonorrhoea, as these signify incident rather than prevalent gonorrhoea. This left 292 couples (584 men) with complete gonorrhoea testing, of whom 36 men $(6.2 \%, 95 \%$ CI 4.4 to 8.4$)$ had throat gonorrhoea and 17 men $(2.9 \%, 95 \%$ CI 1.7 to 4.6$)$ had anal gonorrhoea.

\section{Within-couple genomic relatedness of $\boldsymbol{N}$. gonorrhoeae isolates}

In 11 couples both partners had culture specimens available for WGS. In all 11 couples, both partners had N. gonorrhoeae isolates that were of the same NG-MAST and MLST, and were highly related by core SNP phylogeny, with a median pairwise SNP

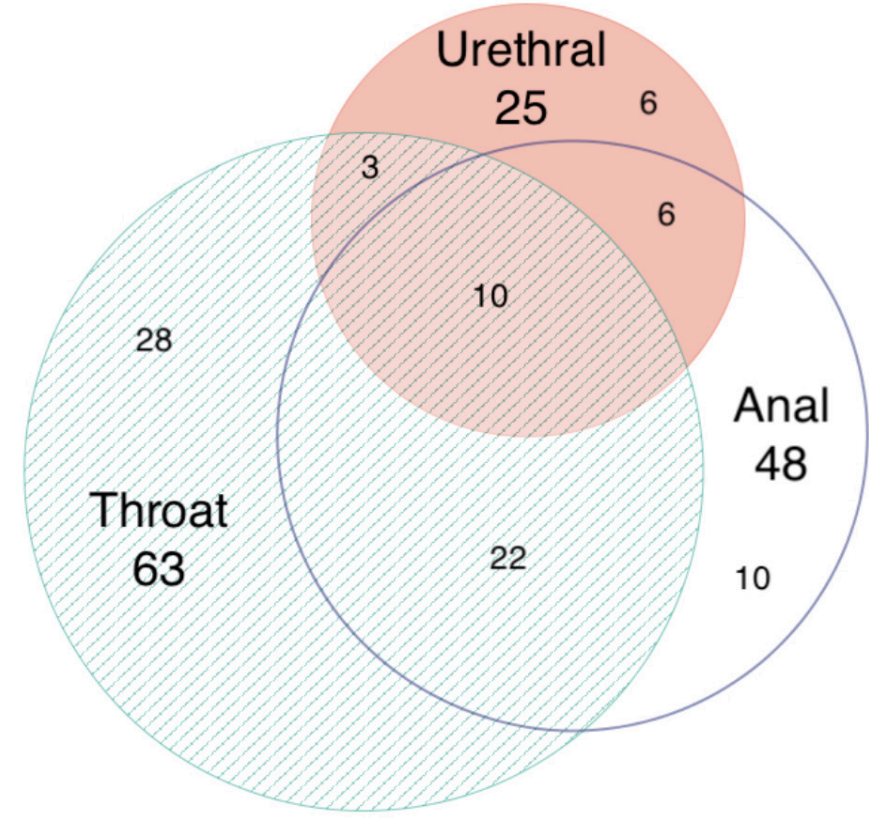

Figure 1 Proportional Venn diagram of anatomical distribution of within-individual gonorrhoea infections in the 120 men who were in couples where both men were tested for gonorrhoea at all three sites (urethra, anus and throat) and in which at least one man tested positive for gonorrhoea. A total of 85 men with gonorrhoea infection at one or more anatomical sites.

distance of 6 (range 0-14 SNPs) between isolates from partner pairs (online supplementary figure $4 \mathrm{~A}$ and $4 \mathrm{~B}$ ).

\section{Gonorrhoea at different anatomical sites}

For men with urethral gonorrhoea, we assessed the proportion whose partner had throat and/or anal gonorrhoea to assess which site is the more likely source of urethral gonorrhoea. Twenty-five men had urethral gonorrhoea, of whom 18 (72\%, 95\% CI 51 to 88) had a partner with throat gonorrhoea and 19 (76\%, 95\% CI 55 to 91 ) had a partner with anal gonorrhoea.

For men with anal gonorrhoea, we assessed the proportion whose partner had throat gonorrhoea. Forty-eight men had anal gonorrhoea, of whom 25 had a partner with throat gonorrhoea (52\%, 95\% CI 37 to 67). When we excluded men with anal gonorrhoea who also had urethral gonorrhoea, 34\% (95\% CI 19 to 53) of 32 men had a partner with throat gonorrhoea. When we excluded men with anal gonorrhoea whose partner had urethral gonorrhoea, $48 \%$ (95\% CI 29 to 68) of 29 men had a partner with throat gonorrhoea (table 2).

\section{Gonorrhoea at the same anatomical sites in couples}

In two couples both partners had urethral gonorrhoea, but in both these couples both men also had both throat gonorrhoea and anal gonorrhoea.

Among the 50 couples where either man had throat gonorrhoea, there were 13 couples (26\% of 50 ; 95\% CI 15 to 40 ) where both men had throat gonorrhoea. To remove the potential contribution of the urethra, we then excluded couples where both men had urethral gonorrhoea, leaving 48 couples where either man had throat gonorrhoea, among whom were 11 couples (23\% of 48 couples; 95\% CI 12 to 37 ) in which both men had throat gonorrhoea (table 3 ).

Among the 33 couples where either man had anal gonorrhoea, there were 15 couples ( $45 \%$ of $33 ; 95 \%$ CI 28 to 64 ) where both 
Table 2 Throat gonorrhoea (NG) positivity in the partners of men with anal gonorrhoea

\begin{tabular}{|c|c|c|c|c|}
\hline \multirow[b]{2}{*}{ Man 1} & \multicolumn{2}{|l|}{ Man 2} & \multirow[b]{2}{*}{ Total } & \multirow{2}{*}{$\begin{array}{l}\% \text { Throat } \\
\text { positive } \\
(95 \% \mathrm{Cl})\end{array}$} \\
\hline & $\begin{array}{l}\text { Throat } \\
\text { NG-negative }\end{array}$ & $\begin{array}{l}\text { Throat } \\
\text { NG-positive }\end{array}$ & & \\
\hline Anal NG-positive & 23 & 25 & 48 & 52 (37 to 67 ) \\
\hline \multicolumn{5}{|c|}{ Couples were excluded if men with anal NG had urethral NG } \\
\hline Anal NG-positive & 21 & 11 & 32 & 34 (19 to 53$)$ \\
\hline \multicolumn{5}{|c|}{ Couples were excluded if men with anal NG had a partner with urethral NG } \\
\hline Anal NG-positive & 15 & 14 & 29 & 48 (29 to 68$)$ \\
\hline
\end{tabular}

NG, Neisseria gonorrhoeae.

men had anal gonorrhoea. To remove the potential contribution of the urethra, we then excluded couples where both men had urethral gonorrhoea, leaving 31 couples where either man had anal gonorrhoea, among whom were 13 couples (42\% of 31 couples; 95\% CI 25 to 61) in which both men had anal gonorrhoea. To remove the potential contribution of the throat, we then excluded couples where either man had throat gonorrhoea, leaving eight couples where either man had anal gonorrhoea, among whom was one couple (13\% of 8 couples; 95\% CI 0 to 53 ) in which both men had anal gonorrhoea (table 3 ).

\section{Urethral gonorrhoea in the 3 months prior to couples' clinic visit}

To assess whether some of the gonorrhoea diagnoses among the included couples may have been a result of recently cleared or treated urethral gonorrhoea in one of the partners, we assessed whether any of the men had recently been diagnosed with urethral gonorrhoea at MSHC. Of the 120 included men, 10 tested for urethral gonorrhoea in the 3 months prior to their clinic visit, of whom none tested positive for gonorrhoea.

Gonorrhoea in men who reported no external sexual partners We conducted a restricted analysis of men who reported no sexual partners other than their regular partner. Of the 120 men, $26(22 \%)$ had no CSPs in the preceding 3 months. Of these, five had urethral gonorrhoea, of whom three had a partner with throat gonorrhoea (60\%, compared with 72\% [95\% CI 51 to 88 ] in the main analysis), and four had a partner with anal gonorrhoea (80\%, compared with 76\% [95\% CI 55 to 91] in the

\begin{tabular}{|c|c|c|c|}
\hline $\begin{array}{l}\text { Couples } \\
\text { where one } \\
\text { man had } \\
\text { throat NG }\end{array}$ & $\begin{array}{l}\text { Couples } \\
\text { where both } \\
\text { men had } \\
\text { throat NG }\end{array}$ & Total couples & $\begin{array}{l}\% \text { of couples where both men } \\
\text { had throat NG } \\
(95 \% \mathrm{Cl})\end{array}$ \\
\hline 37 & 13 & 50 & (15 to 40$)$ \\
\hline \multicolumn{4}{|c|}{ Throat NG positivity when couples were excluded if both men had urethral NG } \\
\hline 37 & 11 & 48 & (12 to 37$)$ \\
\hline $\begin{array}{l}\text { Couples where } \\
\text { one man had } \\
\text { anal NG }\end{array}$ & $\begin{array}{l}\text { Couples where } \\
\text { both men had } \\
\text { anal NG }\end{array}$ & Total couples & $\begin{array}{l}\% \text { of couples where both men } \\
\text { had anal NG } \\
(95 \% \mathrm{Cl})\end{array}$ \\
\hline 18 & 15 & 33 & (28 to 64$)$ \\
\hline \multicolumn{4}{|c|}{ Anal NG positivity when couples were excluded if both men had urethral NG } \\
\hline 18 & 13 & 31 & (25 to 61$)$ \\
\hline \multicolumn{4}{|c|}{ Anal NG positivity when couples were excluded if one or both men had throat NG } \\
\hline 7 & 1 & 8 & (0 to 53$)$ \\
\hline
\end{tabular}

NG, Neisseria gonorrhoeae. main analysis). Of the 26 men with no CSP, 12 had throat gonorrhoea, of whom 4 had a partner with throat gonorrhoea $(33 \%$, compared with 26\% [95\% CI 15 to 40] in the main analysis). Nine men had anal gonorrhoea, of whom four had a partner with throat gonorrhoea (44\%, compared with 52\% [95\% CI 37 to 67$]$ in the main analysis). Hence, the regular partners of men with no external sexual partners had similar positivity of gonorrhoea as seen in our main analysis.

\section{DISCUSSION}

We present the largest study to date of anatomical site-specific gonorrhoea positivity in male couples and the first to use highly sensitive NAAT. Our key finding was that in the absence of urethral infection, when one man in a couple had throat gonorrhoea, his partner commonly had throat gonorrhoea (23\%), and when one man in a couple had anal gonorrhoea his partner commonly had throat gonorrhoea (34\%). These percentages and the lower bounds of their 95\% CIs are substantially higher than would be expected to occur by chance (about $3 \%-6 \%)$, given overall gonorrhoea positivity among the 584 men who presented with their partner (when neither of them had urethral gonorrhoea) was 6\% for throat and 3\% for anal gonorrhoea. Our data are not consistent with the conventional paradigm of gonorrhoea transmission between men, in which the urethra most gonorrhoea transmission is from the urethra to the throat and anus, and vice versa. Instead, our data are consistent with a new paradigm of gonorrhoea transmission, in which the throat plays a central role in transmission to a partner's throat, anus and urethra, presumably through infected saliva. We observed high gonorrhoea positivity in the throat and anus if a man's partner had urethral gonorrhoea. Most cases of urethral gonorrhoea in our study were symptomatic and presented to our clinic within a few of days of developing symptoms. This suggests that the direction of gonorrhoea transmission in these couples was likely from the throat or anus to the urethra. This suggestion is further supported by the finding that none of the participants were diagnosed with urethral gonorrhoea in the 3 months preceding their attendance as a couple. Both the conventional and our new paradigm of gonorrhoea transmission predict high concurrency of urethra/throat and urethra/anus gonorrhoea. However, in the conventional paradigm transmission is bidirectional, whereas in the new paradigm most transmission is unidirectional from the throat and/or anus to the urethra. This assertion by the new paradigm is also supported by the Health in Men study in Australia, where receptive oropenile sex (performing fellatio) was not an independent risk factor for throat gonorrhoea. ${ }^{12}$

We observed moderately high gonorrhoea positivity in the throats of men whose partners had anal gonorrhoea, even after excluding men with urethral gonorrhoea. This is not consistent with the conventional paradigm of gonorrhoea transmission, where the urethra is the source of most gonorrhoea infections at both the throat and the anus. Instead, these data support direct transmission between the throat and the anus. This cross-sectional study cannot determine the direction of transmission, which could be from the throat to the anus or vice versa. We have previously reported that receptive oroanal sex (ie, being rimmed) and the use of partners' saliva as lubricant for penoanal sex were risk factors for anal gonorrhoea, ${ }^{18}$ whereas insertive oroanal sex (ie, performing rimming) was not a risk factor for throat gonorrhoea. ${ }^{13}$ This suggests that the observed throat/anus gonorrhoea concurrency reflects gonorrhoea transmission from the throat to the anus, rather than vice versa. 
We found that when one man in a couple had throat gonorrhoea, there was a high chance that both men in the couple had throat gonorrhoea, even after we excluded couples where both men had urethral gonorrhoea. Under the conventional paradigm, if both men in a couple have throat gonorrhoea, they must both have urethral gonorrhoea as this must be the source of their throat gonorrhoea; hence, our observations are not consistent with the conventional paradigm. Instead, our data suggest that gonorrhoea transmits directly between partners' throats relatively frequently.

We found that when one man in a couple had anal gonorrhoea, there was a high chance that both men in the couple had anal gonorrhoea, even after excluding couples where both men had urethral gonorrhoea, which is not consistent with the conventional paradigm. When we excluded couples where either partner had throat gonorrhoea, concurrent anus/anus gonorrhoea was significantly less frequent. This is consistent with the new transmission paradigm, in which throat gonorrhoea in one partner could transmit to the anus of both partners by way of saliva. Alternatively, direct anus-to-anus transmission of gonorrhoea is possible when men share a dildo, but the receptive use of dildos has previously not been found to be a risk factor for anal gonorrhoea among MSM. ${ }^{19}$

We wish to highlight and address a number of limitations in our study. First, in almost half of couples, at least one man had urethral gonorrhoea that had become symptomatic only a few days prior. Hence our study is biased towards cases of recent transmission to the urethra and does not reflect true population site-specific gonorrhoea prevalence. However, we also summarise gonorrhoea positivity when couples with urethral gonorrhoea were excluded, which should overcome this limitation. A second limitation is that our questionnaire does not define 'your partner', and we have no information on the frequency or nature of their sexual contact, nor whether their last sexual contact was with that partner. Hence our data reflect total transmissibility rather than per-act transmission probability. A third limitation is that our clinic does not routinely collect data about kissing-only partners, so it is possible that men who reported no CSPs had kissing-only partners. A fourth limitation is that we did not adjust our analysis for condom use during anal sex, because the vast majority of participants did not use condoms with their regular partner. A fifth limitation is that we had no data on gonorrhoea infections in sexual partners external to the couples, and hence we cannot exclude that in some of these couples both men may have acquired gonorrhoea from a partner external to the couple, without transmission within the couple. However, a sensitivity analysis of men who reported no contact with external partners in the preceding 3 months showed gonorrhoea concurrency that was similar to our main analysis. Also, phylogenetic analysis of gonorrhoea samples found that $100 \%$ of analysed couples had highly related $N$. gonorrhoeae isolates within the couple, suggesting that gonorrhoea concurrency was likely due to within-couple transmission, consistent with previous work from our group demonstrating high (97\%) within-couple concurrency of N. gonorrhoeae isolates from 34 male couples. ${ }^{20} \mathrm{~A}$ sixth limitation is that, in principle, it is difficult to infer the direction of transmission from cross-sectional data, which is ideally demonstrated by longitudinal analysis. However, we have provided supplementary analyses to provide context and support for our argument of directionality. A final limitation is that we assume that our NAAT assay has high sensitivity at all three anatomical sites, and differential sensitivity would skew our results. NAATs are approved by the USA Food and Drug Administration for detection of gonorrhoea in urine, but not for use at extragenital
Key messages

- This analysis of gonorrhoea in 60 male couples supports a new paradigm of gonorrhoea transmission where throat gonorrhoea is an important driver of transmission.

- Throat gonorrhoea may potentially be transmitted during kissing, oropenile sex, oroanal sex and use of saliva as lubricant during anal sex.

- Public health strategies that aim to control gonorrhoea may need to address these routes of transmission.

sites. However, the NAATs used in our study have been shown to have sensitivity and specificity similar to other assays at both the anus and throat. ${ }^{21}{ }^{22}$ Also, it is not known whether gonorrhoea has a different natural duration at different anatomical sites, which could have affected our results.

In conclusion, our data support a new paradigm of gonorrhoea transmission which suggests that the throat is a major source of gonorrhoea transmission between men, as previously postulated by our group. ${ }^{923}$ This new paradigm proposes that gonorrhoea transmission among MSM occurs mainly through tongue kissing, with onward transmission by oropenile sex, oroanal sex and the use of saliva as lubricant for anal sex. Current public health strategies to control gonorrhoea focus primarily on the use of condoms for penoanal sex, but condoms cannot address the transmission of throat gonorrhoea to partners' throats and anuses. To address these modes of transmission, public health messaging may need to discuss the risk of gonorrhoea transmission during sexual activity that involves saliva. Also, a novel gonorrhoea prevention strategy currently under investigation is the use of antiseptic mouthwash to reduce the prevalence of throat gonorrhoea. ${ }^{24-26}$

\section{Author affiliations \\ ${ }^{1}$ Melbourne Sexual Health Centre, Carlton, Victoria, Australia \\ ${ }^{2}$ Prahran Market Clinic, Prahran, Victoria, Australia \\ ${ }^{3}$ Central Clinical School, Monash University, Prahran, Victoria, Australia \\ ${ }^{4}$ Department of Infectious Diseases, The Alfred Hospital, Melbourne, Victoria, Australia ${ }^{5}$ Melbourne Diagnostic Unit Public Health Laboratory, Peter Doherty Institute, University of Melbourne, Melbourne, Sydney, Australia \\ ${ }^{6}$ The Kirby Institute, UNSW Australia, Kensington, New South Wales, Australia ${ }^{7}$ Melbourne School of Population and Global Health, University of Melbourne, Carlton, Victoria, Australia \\ ${ }^{8}$ Doherty Applied Microbial Genomics, Peter Doherty Institute, Department of Microbiology and Immunology, University of Melbourne, Melbourne, Sydney, Australia}

\section{Handling editor Jackie A Cassell}

Acknowledgements The authors would like to acknowledge A Afrizal for his assistance with data extraction from the MSHC database, and Emile Jasek and Tiffany Phillips for matching partners in couples. Whole genome sequencing was performed at the Microbiological Diagnostic Unit Public Health Laboratory.

Contributors VIC, CKF and EPFC conceived and developed the study. CKF conceived the idea of the new paradigm of transmission for GC. VJC curated the data and conducted the analyses. BPH and DW generated the laboratory diagnostic and whole genome sequence data. DW performed bioinformatic analyses of genomic relatedness. VJC prepared the first draft with assistance from CKF and EPFC. All authors contributed to and approved of the final version.

Funding This work was supported by the Australian National Health and Medical Research Council (NHMRC) programme grant (no: 568971). EPFC is supported by the Early Career Fellowships from the NHMRC (no: 1091226). VJC is supported by a Research Training Scheme Scholarship from the Australian Government's Department of Education and Training. DW is supported by an Early Career Fellowships from the NHMRC (APP1123854). BPH is supported by a Practitioner Fellowship (GNT1105905). VJC had full access to all data in this study and takes final responsibility for the decision to submit for publication. 
Disclaimer The funding sources had no involvement in the design or analysis of this study.

Competing interests None declared.

Patient consent for publication Not required.

Ethics approval Ethics approval was obtained from the Alfred Hospital Ethics Committee, Melbourne, Australia (number 108/15). This was a retrospective analysis of de-identified data; hence, explicit consent to participate in this study was not sought from individual patients. Patients at MSHC are routinely informed that their de-identified data may be used for research purposes and that they can opt out if they object to the use of their data.

Provenance and peer review Not commissioned; externally peer reviewed.

Data sharing statement Reasonable requests for our raw data set will be considered, subject to approval from the Alfred Hospital Ethics Committee. Data from whole genome sequencing of $\mathrm{N}$. gonorrhoeae samples have been uploaded onto the National Center for Biotechnology Information (NCBI), BioProject number PRJNA449254.

\section{REFERENCES}

1 Centers for Disease Control and Prevention. STDs in men who have sex with men, 2016. Available: https://www.cdc.gov/std/stats16/msm.htm [Accessed 3 Mar 2018].

2 The Kirby Institute. HIV, viral hepatitis and sexually transmissible infections in Australia: the Kirby Institute, UNSW Australia, 2017. Available: https://kirby.unsw. edu.au/sites/default/files/kirby/report/SERP_Annual-Surveillance-Report-2017_ compressed.pdf [Accessed 3 Mar 2018]

3 Blomquist PB, Miari VF, Biddulph JP, et al. Is gonorrhea becoming untreatable? Future Microbiol 2014;9:189-201.

4 World Health Organization. Global action plan to control the spread and impact of antimicrobial resistance in Neisseria gonorrhoeae: World Health organization, 2012. Available: http://apps.who.int/iris/bitstream/handle/10665/44863/9789241503501 eng.pdf; jsessionid=3B44C236F95271 C8E3159480C9AF020E? sequence $=1$ [Accessed 3 Mar 2018].

5 Centers for Disease Control and Prevention. Antibiotic resistance threats in the United States: U.S. department of health and Human services, 2013. Available: https://www. cdc.gov/drugresistance/threat-report-2013/pdf/ar-threats-2013-508.pdf [Accessed 3 Mar 2018].

6 Ryder N, Lockart IG, Bourne C. Is screening asymptomatic men who have sex with men for urethral gonorrhoea worthwhile? Sex. Health 2010;7:90-1.

7 Ong JJ, Fethers K, Howden BP, et al. Asymptomatic and symptomatic urethral gonorrhoea in men who have sex with men attending a sexual health service. Clin Microbiol Infect 2017;23:555-9.

8 Fairley CK, Chow EPF, Hocking JS. Early presentation of symptomatic individuals is critical in controlling sexually transmissible infections. Sex. Health 2015;12:181-2.

9 Fairley CK, Hocking JS, Zhang L, et al. Frequent transmission of gonorrhea in men who have sex with men. Emerg Infect Dis 2017;23:102-4.

10 Bro-Jorgensen A, Jensen T. Gonococcal pharyngeal infections. Report of 110 cases. Br J Vener Dis 1973;49:491-9.
11 Willmott FE. Transfer of gonococcal pharyngitis by kissing? Br J Vener Dis 1974:50:317-8.

12 Templeton DJ, Jin F, McNally LP, et al. Prevalence, incidence and risk factors for pharyngeal gonorrhoea in a community-based HIV-negative cohort of homosexual men in Sydney, Australia. Sex Transm Infect 2010;86:90-6.

13 Cornelisse VJ, Walker S, Phillips T, et al. Risk factors for oropharyngeal gonorrhoea in men who have sex with men: an age-matched case-control study. Sex Transm Infect 2018:94:359-64.

14 Chow EPF, Priest D, Cornelisse VJ, et al. Kissing but not sex is the strongest risk factor for oropharyngeal gonorrhoea in men who have sex with men: a cross-sectional survey. Edinburgh, Scotland: Fourth Joint Conference of the British Association for Sexual Health and HIV (BASHH) with the British HIV Association (BHIVA), 2018.

15 Vodstrcil LA, Hocking JS, Cummings R, et al. Computer assisted self interviewing in a sexual health clinic as part of routine clinical care; impact on service and patient and clinician views. PLOS ONE 2011:6:e18456.

16 Templeton DJ, Read P, Varma R, et al. Australian sexually transmissible infection and HIV testing guidelines for asymptomatic men who have sex with men 2014: a review of the evidence. Sex. Health 2014;11:217-29.

17 Morisette JT, Khorram S. Exact binomial confidence interval for proportions. Photogrammetr Eng Remote Sens 1998;64:281-3.

18 Chow EPF, Cornelisse VJ, Read TRH, et al. Saliva use as a lubricant for anal sex is a risk factor for rectal gonorrhoea among men who have sex with men, a new public health message: a cross-sectional survey. Sex Transm Infect 2016;92:532-6.

19 Jin F, Prestage GP, Mao L, et al. Incidence and risk factors for urethral and anal gonorrhoea and Chlamydia in a cohort of HIV-negative homosexual men: the Health in Men Study. Sex Transm Infect 2007:83:113-9.

20 Kwong JC, Chow EPF, Stevens K, et al. Whole-genome sequencing reveals transmission of gonococcal antibiotic resistance among men who have sex with men: an observational study. Sex Transm Infect 2018;94:151-7.

21 Bachmann LH, Johnson RE, Cheng $\mathrm{H}$, et al. Nucleic acid amplification tests for diagnosis of Neisseria gonorrhoeae and Chlamydia trachomatis rectal infections. J Clin Microbiol 2010;48:1827-32.

22 Bachmann LH, Johnson RE, Cheng $\mathrm{H}$, et al. Nucleic acid amplification tests for diagnosis of Neisseria gonorrhoeae oropharyngeal infections. J Clin Microbiol 2009;47:902-7.

23 Zhang L, Regan DG, Chow EPF, et al. Neisseria gonorrhoeae transmission among men who have sex with men: an anatomical site-specific mathematical model evaluating the potential preventive impact of mouthwash. Sex Transm Dis 2017;44:586-92.

24 Cornelisse VJ, Fairley CK, Walker S, et al. Adherence to, and acceptability of, Listerine $\mathbb{}$ mouthwash as a potential preventive intervention for pharyngeal gonorrhoea among men who have sex with men in Australia: a longitudinal study. Sex Health 2016;13:494-6.

25 Chow EPF, Walker S, Hocking JS, et al. A multicentre double-blind randomised controlled trial evaluating the efficacy of daily use of antibacterial mouthwash against oropharyngeal gonorrhoea among men who have sex with men: the omega (oral mouthwash use to eradicate gonorrhoea) study protocol. BMC Infect Dis 2017;17.

26 Chow EP, Howden BP, Walker S, et al. Antiseptic mouthwash against pharyngeal Neisseria gonorrhoeae: a randomised controlled trial and an in vitro study. Sex Transm Infect 2017;93:88-93. 